\title{
Quantum-metric contribution to the pair mass in spin-orbit-coupled Fermi superfluids
}

\author{
M. Iskin \\ Department of Physics, Koç University, Rumelifeneri Yolu, 34450 Sarlyer, Istanbul, Turkey
}

(Received 23 January 2018; revised manuscript received 5 March 2018; published 28 March 2018)

\begin{abstract}
As a measure of the quantum distance between Bloch states in the Hilbert space, the quantum metric was introduced to solid-state physics through the real part of the so-called geometric Fubini-Study tensor, the imaginary part of which corresponds to the Berry curvature measuring the emergent gauge field in momentum space. Here, we first derive the Ginzburg-Landau theory near the critical superfluid transition temperature and then identify and analyze the geometric effects on the effective mass tensor of the Cooper pairs. By showing that the quantum-metric contribution accounts for a sizable fraction of the pair mass in a surprisingly large parameter regime throughout the BCS-Bose-Einstein condensate crossover, we not only reveal the physical origin of its governing role in the superfluid density tensor but also hint at its plausible roles in many other observables.
\end{abstract}

DOI: 10.1103/PhysRevA.97.033625

\section{INTRODUCTION}

Along with the Berry curvature, the quantum metric encodes some of the elusive quantum geometrical properties not only of the modern solid-state materials and condensed-matter systems (e.g., the quantum and spin Hall states, and topological insulators and superconductors [1-5]) but also of the photonics systems (e.g., the gyromagnetic photonic crystals, coupled resonators and waveguides, bianisotropic metamaterials, and quasicrystals [6,7]) and of the quantum gases (e.g., the Hofstadter and Haldane models, and topological and geometrical charge pumps [8-12]). Even though both the Berry curvature and the quantum metric characterize by definition [13] the local momentum-space geometry of the underlying Bloch states, they may also be linked with the global properties of the system in somewhat peculiar ways. For instance, the topological Chern invariant of a quantum Hall system is simply determined by an integration of the Berry curvature over its entire Brillouin zone, controlling the Hall conductivity [1-5]. Likewise, in the context of multiband superconductors, in addition to the conventional intraband contribution determined by the electronic spectra of the Bloch bands, the superfluid (SF) weight has an additional contribution coming from the so-called geometric interband processes [14]. In the particular case of isolated (but not necessarily flat) band superconductors, the interband contribution is determined by an integration that depends explicitly on the quantum metric of the isolated Bloch band, and it is precisely because of the presence of this contribution that superfluidity prevails in the isolated flat-band limit for which the intraband contribution necessarily vanishes $[15,16]$.

Despite a long history of interdisciplinary interest on a variety of physical phenomena controlled by the Berry curvature [1-12], nature has not so far been as generous to the quantum-metric effects, given their relatively more recent and very limited applications in condensed-matter physics theory [14-31]. For instance, the quantum metric of the noninteracting helicity bands, which are characterized by the projection of the spin onto the direction of momentum in the presence of spin-orbit coupling (SOC), is proposed to be responsible for up to a quarter of the total SF density [31]. This is surely a very timely offering motivated by the recent creation of Rashba SOC with ultracold Bose and Fermi gases [32-35]. Inspired by these developments, here we first identify and then show that the quantum-metric contribution accounts for a sizable fraction of the effective mass tensor of the Cooper pairs in a surprisingly large parameter regime throughout the BCS-Bose-Einstein condenstate (BEC) crossover. This finding not only reveals the physical origin of the quantum metric's governing role in the SF density tensor [14-16,29,31] but also hints at its plausible roles in many other observables including sound velocity, atomic compressibility, spin susceptibility, etc., all of which depend explicitly on the pair mass. Furthermore, even though most of our analysis is specified for the spin-orbit-coupled systems, our starting Hamiltonian is quite generic and may find direct applications in other two-band SFs as well [36,37].

\section{SINGLE-PARTICLE HAMILTONIAN AND TWO-BODY PROBLEM}

Having a two-component or pseudospin- $1 / 2$ Fermi gas with a generic SOC in mind, here we consider a class of singleparticle problems that are described by the wave equation $H_{\mathbf{k}}|s \mathbf{k}\rangle=\epsilon_{\mathbf{s} \mathbf{k}}|s \mathbf{k}\rangle$, for which the noninteracting Hamiltonian density $H_{\mathbf{k}}=\epsilon_{\mathbf{k}} \sigma_{0}+\mathbf{d}_{\mathbf{k}} \cdot \sigma$ in $\mathbf{k}$ space leads to a couple of energy (helicity) bands that are indexed by $s= \pm$. Thus, the energy spectra of the noninteracting particles can be expressed as $\epsilon_{s \mathbf{k}}=\epsilon_{\mathbf{k}}+s d_{\mathbf{k}}$, along, respectively, with the following energy eigenstates $|s \mathbf{k}\rangle^{\mathrm{T}}=\left(-d_{\mathbf{k}}^{x}+i d_{\mathbf{k}}^{y}, d_{\mathbf{k}}^{z}-s d_{\mathbf{k}}\right) / \sqrt{2 d_{\mathbf{k}}\left(d_{\mathbf{k}}-s d_{\mathbf{k}}^{z}\right)}$, where $\mathrm{T}$ is the transpose operator. Here, $\mathbf{k}=\sum_{i} k_{i} \widehat{i}$ is the wave vector, $\epsilon_{\mathbf{k}}=k^{2} /(2 m)$ with $\hbar \rightarrow 1$ is taken (in this paper) as the usual quadratic dispersion of a free particle, $\sigma_{0}$ is the $2 \times 2$ identity matrix, $\mathbf{d}_{\mathbf{k}}=\sum_{i} d_{\mathbf{k}}^{i} \hat{\boldsymbol{i}}$ with the magnitude $d_{\mathbf{k}}=\left|\mathbf{d}_{\mathbf{k}}\right|$ is the SOC field, and $\boldsymbol{\sigma}=\sum_{i} \sigma_{i} \hat{\boldsymbol{i}}$ is a vector of Pauli spin matrices. Note that $\widehat{i}$ is the unit vector along the $i$ direction in such a way that $d_{\mathbf{k}}^{i}=\alpha_{i} k_{i}$ corresponds to a Rashba SOC when $\alpha_{x}=\alpha_{y}=\alpha$ and $\alpha_{z}=0$, and to a Weyl SOC when $\alpha_{x}=\alpha_{y}=\alpha_{z}=\alpha$. We choose $\alpha \geqslant 0$ without losing generality. 
In the presence of an attractive and short-ranged twobody interaction between an $\uparrow$ and a $\downarrow$ particle, its strength $U \geqslant 0$ may be linked to the two-body binding energy $\epsilon_{b} \leqslant 0$ in vacuum via the relation $2 / U=\sum_{s \mathbf{k}} 1 /\left(2 \epsilon_{s \mathbf{k}}+\right.$ $\left.\epsilon_{\mathrm{th}}-\epsilon_{b}\right)$, where $\epsilon_{\mathrm{th}}=m \alpha^{2}$ is the energy threshold for the formation of the two-body bound states. In addition, for a three-dimensional (3D) Fermi gas, the theoretical parameter $U$ may also be eliminated in favor of the experimentally relevant $s$-wave scattering length $a_{s}$ via the usual relation, $1 / U=-m V /\left(4 \pi a_{s}\right)+\sum_{\mathbf{k}} 1 /\left(2 \epsilon_{\mathbf{k}}\right)$, where $V$ is the volume. While the combination of these relations leads to an implicit equation of the form $1 /\left(m \alpha a_{s}\right)=\sqrt{1+\left|\epsilon_{b}\right| /\left(m \alpha^{2}\right)}-$ $\ln \left(\sqrt{1+m \alpha^{2} /\left|\epsilon_{b}\right|}+\sqrt{m \alpha^{2} /\left|\epsilon_{b}\right|}\right)$ for a Rashba SOC, it leads to $\left|\epsilon_{b}\right|=1 /\left(2 m a_{s}^{2}\right)+m \alpha^{2} \mp \sqrt{1 /\left(4 m^{2} a_{s}^{4}\right)+\alpha^{2} / a_{s}^{2}}$ for a Weyl SOC where the $\mp$ sign is for the $a_{s} \lessgtr 0$ region. Note that by setting $\left|a_{s}\right| \rightarrow \infty$ in these implicit expressions, we find $\epsilon_{b} \approx-0.44 m \alpha^{2}$ for the Rashba SOC and $\epsilon_{b}=-m \alpha^{2}$ for the Weyl SOC at unitarity. For a 2D Fermi gas, however, eliminating $g$ via the usual relation $1 / U=\sum_{\mathbf{k}} 1 /\left(2 \epsilon_{\mathbf{k}}-\epsilon_{s b}\right)$, we find $\left|\epsilon_{s b}\right|=\left(\left|\epsilon_{b}\right|+\right.$ $\left.m \alpha^{2}\right) \exp \left[-2 \sqrt{m \alpha^{2} /\left|\epsilon_{b}\right|} \arctan \left(\sqrt{m \alpha^{2} /\left|\epsilon_{b}\right|}\right)\right]$ for a Rashba SOC.

\section{MANY-BODY PROBLEM AND GINZBURG-LANDAU THEORY}

Once the formation of all sorts of Cooper pairs, e.g., consisting of $\uparrow$ particles with $\mathbf{k}+\mathbf{q} / 2$ momentum and $\downarrow$ particles with $-\mathbf{k}+\mathbf{q} / 2$ momentum, is taken into account, the resultant effective action may be approximated as $S_{\text {eff }} \approx S_{0}+S_{\text {Gauss }}$, where the first (second) term is the saddle-point (Gaussian fluctuation) contribution coming from the stationary (nonstationary) Cooper pairs with zero (finite) center-of-mass momentum q [38-40]. Assuming an equal number of particles for the pseudospin components, $S_{0}=\Omega_{m f} / T$ is determined by the mean-field thermodynamic potential $\Omega_{m f}=-T \sum_{s \mathbf{k}} \ln [1+$ $\left.\exp \left(-E_{s \mathbf{k}} / T\right)\right]+\sum_{s \mathbf{k}}\left(\xi_{s \mathbf{k}}-E_{s \mathbf{k}}\right) / 2+\Delta^{2} / U[40-45]$, where $T$ is the temperature with $k_{B} \rightarrow 1$ being the Boltzmann constant, $\xi_{s \mathbf{k}}=\epsilon_{s \mathbf{k}}-\mu$ is the shifted dispersion for the $s$ helicity band with $\mu$ being the chemical potential, and $E_{s \mathbf{k}}=$ $\left(\xi_{s \mathbf{k}}^{2}+\Delta^{2}\right)^{1 / 2}$ is the spectrum of the quasiparticles for the corresponding helicity band. Here, the BCS mean-field $\Delta=$ $U\left\langle\psi_{\uparrow \mathbf{k}} \psi_{\downarrow-\mathbf{k}}\right\rangle$ is taken as a real order parameter without losing generality, where $\langle\cdots\rangle$ denotes a thermal average over the pair-annihilation operator. The mean-field self-consistency equations for $\Delta$ and $\mu$ are $1 / U=\sum_{s \mathbf{k}} \mathcal{X}_{s \mathbf{k}} /\left(4 E_{s \mathbf{k}}\right)$ and $N_{\mathrm{mf}}=$ $\sum_{s \mathbf{k}}\left[1 / 2-\xi_{s \mathbf{k}} \mathcal{X}_{s \mathbf{k}} /\left(2 E_{s \mathbf{k}}\right)\right]$, where $\mathcal{X}_{s \mathbf{k}}=\tanh \left[E_{s \mathbf{k}} /(2 T)\right]$ is a thermal factor.

In order to derive the Ginzburg-Landau theory describing the low-energy dynamics of the order parameter near the critical SF transition temperature $T_{c}$, we restrict our analysis to its vicinity and calculate the Gaussian contribution $S_{\text {Gauss }}$ to the action by expanding the order parameter field around $\Delta=0$ up to quadratic order in the fluctuations [38-40]. This leads to $S_{\text {Gauss }}=(1 / T) \sum_{\ell \mathbf{q}} \mathcal{L}_{\ell \mathbf{q}}^{-1}\left|\Lambda_{\ell \mathbf{q}}\right|^{2}$, where $\Lambda_{\ell \mathbf{q}}$ is the fluctuation field with $\mathbf{q}$ being the momentum of the Cooper pairs and $\nu_{\ell}=2 \pi T \ell$ being the bosonic Matsubara frequency, and

$$
\mathcal{L}_{\ell \mathbf{q}}^{-1}=\frac{1}{U}-\frac{1}{8} \sum_{s s^{\prime} \mathbf{k}} \frac{\mathcal{X}_{s+}+\mathcal{X}_{s^{\prime}-}}{\xi_{s+}+\xi_{s^{\prime}-}-i \nu_{\ell}}\left(1-s s \widehat{\mathbf{d}}_{+} \cdot \widehat{\mathbf{d}}_{-}\right)
$$

is the inverse fluctuation propagator [40-45]. Here, $\mathcal{X}_{s \pm}=$ $\tanh \left[\xi_{s \pm} /(2 T)\right]$ is a shorthand notation with $\xi_{s \pm}=\xi_{s, \pm \mathbf{k}+\mathbf{q} / 2}$ for the shifted dispersions, and $\widehat{\mathbf{d}}_{ \pm}$is for the unit vectors along the SOC fields $\mathbf{d}_{ \pm \mathbf{k}+\mathbf{q} / 2}$. By further expanding the inverse propagator at low momentum (up to second-order) and low frequency (up to first order in $\omega$ after the analytical continuation $\left.i v_{\ell} \rightarrow \omega+i 0^{+}\right)$,

$$
\mathcal{L}_{\omega \mathbf{q}}^{-1} \approx a(T)+\frac{1}{2} \sum_{i j} c_{i j} q_{i} q_{j}-d_{0} \omega+\cdots,
$$

we eventually arrive at the celebrated time-dependent Ginzburg-Landau equation [38-40]. More precisely, the microscopic parameters $a(T), d_{0}$, and $c_{i j}$ correspond to the coefficients of its quadratic terms, describing numerous properties of the system. We note that the quartic term describes the pair-pair interactions, and it is not of particular interest within the scope of this paper. For instance, the $T$-dependent coefficient $a(T)=1 / U-\sum_{s \mathbf{k}} \mathcal{X}_{s \mathbf{k}} /\left(4 \xi_{s \mathbf{k}}\right)$ gives precisely the Thouless criterion $a\left(T_{c}\right)=0$ for $T_{c}$, and the complex number $d_{0}=$ $\sum_{s \mathbf{k}} \mathcal{X}_{s \mathbf{k}} /\left(8 \xi_{s \mathbf{k}}^{2}\right)+i \pi \lim _{\omega \rightarrow 0^{+}} \sum_{s \mathbf{k}} \mathcal{X}_{s \mathbf{k}} \delta\left(2 \xi_{s \mathbf{k}}-\omega\right) /\left(4 \xi_{s \mathbf{k}}\right)$ is the coefficient of the time-dependent term with $\delta(x)$ being the Dirac delta function. While its nonzero imaginary part for $\mu \geqslant-m \alpha^{2} / 2$ reflects the finite lifetime of the many-body bound states, i.e., due to their instability towards decaying into the two-body continuum, its purely real value for $\mu<-m \alpha^{2} / 2$ reflects the eventual stability of the two-body bound states that are propagating in time with long lifetimes $[39,40]$.

Most important of all, we notice that the coefficient of the kinetic term $c_{i j}=c_{i j}^{\text {intra }}+c_{i j}^{\text {inter }}$ has two contributions originating from physically distinct mechanisms, i.e.,

$$
\begin{gathered}
c_{i j}^{\text {intra }}=\sum_{s \mathbf{k}}\left(\frac{\mathcal{X}_{s \mathbf{k}}}{16 \xi_{s \mathbf{k}}^{2}}-\frac{\mathcal{Y}_{s \mathbf{k}}}{32 T \xi_{s \mathbf{k}}}\right) \frac{\partial^{2} \xi_{s \mathbf{k}}}{\partial k_{i} \partial k_{j}} \\
+\sum_{s \mathbf{k}} \frac{\mathcal{X}_{s \mathbf{k}} \mathcal{Y}_{s \mathbf{k}}}{32 T^{2} \xi_{s \mathbf{k}}} \frac{\partial \xi_{s \mathbf{k}}}{\partial k_{i}} \frac{\partial \xi_{s \mathbf{k}}}{\partial k_{j}}, \\
c_{i j}^{\text {inter }}=-\sum_{s \mathbf{k}} \frac{s d_{\mathbf{k}} \mathcal{X}_{s \mathbf{k}}}{4 \xi_{\mathbf{k}} \xi_{s \mathbf{k}}} g_{\mathbf{k}}^{i j},
\end{gathered}
$$

where $\mathcal{Y}_{s \mathbf{k}}=\operatorname{sech}^{2}\left[\xi_{s \mathbf{k}} /(2 T)\right]$ is a thermal factor, and $g_{\mathbf{k}}^{i j}=$ $(1 / 2) \lim _{\mathbf{q} \rightarrow \mathbf{0}} \partial^{2}\left(\widehat{\mathbf{d}}_{+} \cdot \widehat{\mathbf{d}}_{-}\right) /\left(\partial q_{i} \partial q_{j}\right)$ or equivalently $g_{\mathbf{k}}^{i j}=$ $\left(\partial \widehat{\mathbf{d}}_{\mathbf{k}} / \partial k_{i}\right) \cdot\left(\partial \widehat{\mathbf{d}}_{\mathbf{k}} / \partial k_{j}\right) / 2$ is solely controlled by the details of the SOC field. Here, $c_{i j}=c_{j i}$ is necessarily symmetric in its indices. The former contribution $c_{i j}^{\text {intra }}$ has precisely the conventional form arising from the tunneling of the particles within the individual helicity bands, hence its name intraband. However, the latter contribution $c_{i j}^{\text {inter }}$ is due to the tunneling of the particles between the helicity bands, hence its name interband. Next we show that the interhelicity contribution has its roots in the quantum geometry of the underlying $\mathbf{k}$ space, making its revelation one of our primary findings in this work.

\section{QUANTUM METRIC AND BERRY CURVATURE}

First, let us recall that, given a noninteracting multiband Hamiltonian density $H_{\mathbf{k}}$, the quantum metric $g_{n \mathbf{k}}^{i j}$ and the Berry curvature $F_{n \mathbf{k}}^{i j}$ of a given Bloch band $n$ are determined by the real and imaginary parts of the so-called quantum geometric 
tensor $Q_{n \mathbf{k}}^{i j}=g_{n \mathbf{k}}^{i j}-(i / 2) F_{n \mathbf{k}}^{i j}$ of the projected Hilbert space defined by $\left(\partial\langle n \mathbf{k}| / \partial k_{i}\right)(\mathbb{I}-|n \mathbf{k}\rangle\langle n \mathbf{k}|)\left(\partial|n \mathbf{k}\rangle / \partial k_{j}\right) \quad[3,13]$. Here, the completeness relation is not for the entire Hilbert space, but is limited to the subspace of $\mathbf{k}$ states, in such a way that $\mathbb{I}=\sum_{n^{\prime}}\left|n^{\prime} \mathbf{k}\right\rangle\left\langle n^{\prime} \mathbf{k}\right|$ with $n^{\prime}$ summing over all of the available bands. Alternatively, it is numerically much more practical to implement the elements of the $Q_{n \mathbf{k}}^{i j}$ tensor in terms of the derivatives of the Hamiltonian density as follows: $Q_{n \mathbf{k}}^{i j}=$ $\sum_{n^{\prime}\{\neq n\}}\left\langle n \mathbf{k}\left|\left(\partial H_{\mathbf{k}} / \partial k_{i}\right)\right| n^{\prime} \mathbf{k}\right\rangle\left\langle n^{\prime} \mathbf{k}\left|\left(\partial H_{\mathbf{k}} / \partial k_{j}\right)\right| n \mathbf{k}\right\rangle /\left(\epsilon_{n \mathbf{k}}-\epsilon_{n^{\prime} \mathbf{k}}\right)^{2}$. This is because, since the eigenstates $|n \mathbf{k}\rangle$ for a given $\mathbf{k}$ are determined up to a random phase factor in a computer program, further computation of the derivatives in the original definition produces indeterminate factors. Such a numerical ambiguity is clearly avoided by the latter formulation. For the case of two bands that are described by our generic single-particle problem $H_{\mathbf{k}}|s \mathbf{k}\rangle=\epsilon_{s \mathbf{k}}|s \mathbf{k}\rangle$, it can be shown analytically that, while the quantum metrics $g_{s \mathbf{k}}^{i j}=\left(\partial \widehat{\mathbf{d}}_{\mathbf{k}} / \partial k_{i}\right) \cdot\left(\partial \widehat{\mathbf{d}}_{\mathbf{k}} / \partial k_{j}\right) / 4$ are identical for both bands, the Berry curvatures are exactly opposite $F_{s \mathbf{k}}^{i j}=s F_{\mathbf{k}}^{i j}$ of each other with $F_{\mathbf{k}}^{i j}=\left[\left(\partial \widehat{\mathbf{d}}_{\mathbf{k}} / \partial k_{i}\right) \times\left(\partial \widehat{\mathbf{d}}_{\mathbf{k}} / \partial k_{j}\right)\right] \cdot \widehat{\mathbf{d}}_{\mathbf{k}} / 2$. Thus, while $g_{s \mathbf{k}}^{i j}$ is a symmetric tensor, $F_{s \mathbf{k}}^{i j}$ is an antisymmetric one. In addition, the components of the latter tensor are determined by the those of the former up to a $\mathbf{k}$-dependent sign in the following way: $\left|F_{\mathbf{k}}^{i j}\right|=\left(g_{\mathbf{k}}^{i i} g_{\mathbf{k}}^{j j}-g_{\mathbf{k}}^{i j} g_{\mathbf{k}}^{j i}\right)^{1 / 2}$, where $g_{\mathbf{k}}^{i j}=\sum_{s} g_{s \mathbf{k}}^{i j}$ is the total quantum metric of the helicity bands appearing explicitly in Eq. (4).

Given the microscopic coefficients $a(T), d_{0}$, and $c_{i j}$ of the Ginzburg-Landau theory derived above, an effective GrossPitaevskii theory for the corresponding Bose gas of weakly interacting pairs can be obtained upon the rescaling of the fluctuation field as $\Psi_{\omega \mathbf{q}}=\sqrt{d_{0}} \Lambda_{\omega \mathbf{q}}$. Note that this particular choice transforms the coefficient of the time-dependent term to that of the Schrodinger one; i.e., it becomes $i \hbar \partial \Psi_{t \mathbf{r}} / \partial t$ in real space $\mathbf{r}$ and time $t[39,40]$. This identification implies that the effective mass tensor of the Cooper pairs is simply given by $\left(m_{B}^{-1}\right)^{i j}=c_{i j} / d_{0}$ for any given set of parameters, demonstrating the existence of a quantum geometric contribution to the pair mass in general. As the numerical calculation of these coefficients necessitates the self-consistent solutions for $T_{c}$ and $\mu$ in general, one needs to go beyond the mean-field approximation and include the Gaussian fluctuation contribution $N_{\text {Gauss }}$ to the number equation as the minimal prescription for a reliable description. For its simplicity, next we restrict our analysis to the weakly interacting BEC limit of small bosonic molecules, whose analytically tractable nature already illustrates quite convincingly the relative importance of the geometric effects without any reliance on heavy numerics.

\section{FATE OF COOPER PAIRS IN THE MOLECULAR BOSE GAS LIMIT}

Since this limit is achieved when $\mu<-m \alpha^{2} / 2$ and $|\mu| \gg$ $T_{c}$, i.e., $\xi_{s \mathbf{k}} / T \rightarrow \infty$ for every $\mathbf{k}$, we may simply set $\mathcal{X}_{s \mathbf{k}} \rightarrow 1$ and $\mathcal{Y}_{s \mathbf{k}} \rightarrow 0$ in this limit, offering a tremendous simplification of the problem. For instance, eliminating $U$ in favor of $\epsilon_{b}$ and using the Thouless condition $a\left(T_{c}\right)=0$, with $a(T)=$ $1 / U-\sum_{s \mathbf{k}} 1 /\left(4 \xi_{s \mathbf{k}}\right)$, we find $|\mu|=\left(m \alpha^{2}+\left|\epsilon_{b}\right|\right) / 2$ at $T_{c}$ for the molecular Bose gas. In addition, the rest of the coefficients

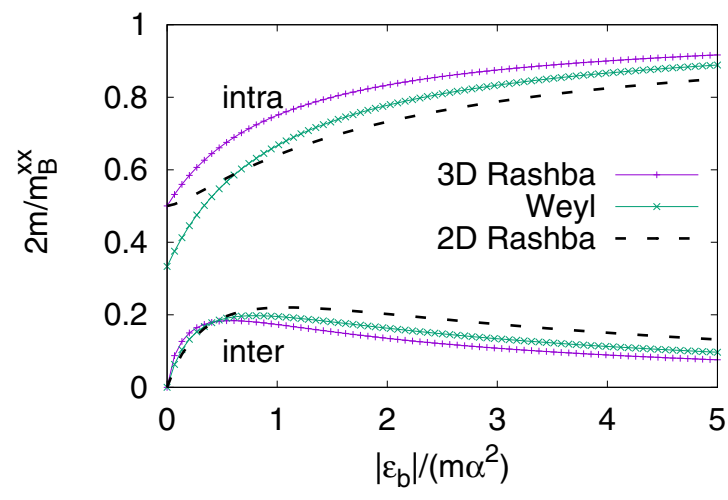

FIG. 1. The intrahelicity and interhelicity contributions to the effective mass of the Cooper pairs $2 m / m_{B}^{x x}=2 m c_{x x} / d_{0}$ are shown for a molecular Bose gas near $T_{c}$. Since the weakly interacting BEC limit is characterized by $\mu<-m \alpha^{2} / 2$ and $|\mu|=\left(m \alpha^{2}+\left|\epsilon_{b}\right|\right) / 2 \gg T_{c}$, it is possible to achieve this limit by simply increasing $\alpha$ no matter how small $\left|\epsilon_{b}\right| \neq 0$ or equivalently interaction strength $U \neq 0$ is. Note that the peak value of the quantum-metric contribution coincides nearly with the location of the unitarity in a 3D system, e.g., $\left|a_{s}\right| \rightarrow \infty$ when $\left|\epsilon_{b}\right| \approx 0.44 m \alpha^{2}$ for the Rashba SOC and $\left|\epsilon_{b}\right|=m \alpha^{2}$ for the Weyl SOC as discussed in the text.

reduce to $d_{0}=\sum_{s \mathbf{k}} 1 /\left(8 \xi_{s \mathbf{k}}^{2}\right)$ for the time-dependent term, and $c_{i j}^{\text {intra }}=\sum_{s \mathbf{k}}\left[\partial^{2} \xi_{s \mathbf{k}} /\left(\partial k_{i} \partial k_{j}\right)\right] /\left(16 \xi_{s \mathbf{k}}^{2}\right)$ for the intrahelicity contribution and $c_{i j}^{\text {inter }}=-\sum_{s \mathbf{k}} s d_{\mathbf{k}} g_{\mathbf{k}}^{i j} /\left(4 \xi_{\mathbf{k}} \xi_{s \mathbf{k}}\right)$ for the interhelicity contribution to the kinetic term. Note that the derivative $\partial^{2} \xi_{s \mathbf{k}} /\left(\partial k_{i} \partial k_{j}\right)=\partial^{2} \xi_{\mathbf{k}} /\left(\partial k_{i} \partial k_{j}\right)+s \partial^{2} d_{\mathbf{k}} /\left(\partial k_{i} \partial k_{j}\right)$ of the helicity spectrum appearing in the intrahelicity contribution may also be expressed in terms of the quantum metric as follows: $\partial^{2} d_{\mathbf{k}} /\left(\partial k_{i} \partial k_{j}\right)=2 d_{\mathbf{k}} g_{\mathbf{k}}^{i j}+\widehat{\mathbf{d}}_{\mathbf{k}} \cdot \partial^{2} \mathbf{d}_{\mathbf{k}} /\left(\partial k_{i} \partial k_{j}\right)$. While the latter term vanishes for typical SOCs, the former is in direct competition with the interhelicity contribution due to the difference in their overall signs. This competition is best seen in Fig. 1, where we find that the intrahelicity (inter-helicity) term has a negative (positive) contribution to the usual result $2 m / m_{B}=1$, i.e., when $\alpha \rightarrow 0$, in all cases considered in this paper.

Prior to presenting our detailed analysis for these coefficients, we note in passing that $c_{i j}=c_{i i} \delta_{i j}$, and hence $m_{B}^{i j}$ is a diagonal tensor for the Rashba and Weyl SOCs that are considered in this paper. In addition, the critical SF transition temperature $T_{c}$ of the resultant weakly interacting molecular Bose gas in 3D is well approximated by the critical BEC temperature of a noninteracting Bose gas determined by the usual number equation $N_{B}=\sum_{\mathbf{q}} 1 /\left[\exp \left(\epsilon_{B \mathbf{q}} / T_{C}\right)-1\right]$. Here, $N_{B}$ is precisely the pole contribution of the Gaussian fluctuations characterized by the propagator given in Eq. (1) [38-40]. Thus, by plugging $\epsilon_{B \mathbf{q}}=\sum_{i} q_{i}^{2} /\left(2 m_{B}^{i i}\right)$ for the low-energy spectrum of our pairs, we find $T_{c}=2 \pi\left\{N_{B} /\left[V \sqrt{m_{B}^{x x} m_{B}^{y y} m_{B}^{z z}} \zeta(3 / 2)\right]\right\}^{2 / 3}$, with $\zeta(3 / 2) \approx 2.61$ the Riemann zeta function. Furthermore, by setting $N_{B} \approx N / 2$ for the pairs with $N=k_{F}^{3} V /\left(3 \pi^{2}\right)$ and the Fermi energy $\epsilon_{F}=k_{F}^{2} /(2 m)$, we eventually obtain $T_{c} / \epsilon_{F} \approx 0.218\left[2 m\left(c_{x x} c_{y y} c_{z z}\right)^{1 / 3} / d_{0}\right]$ for our molecular Bose gas. In a weakly interacting molecular 2D Bose gas, however, $T_{c}$ is determined by an analogy with the BerezinskiiKosterlitz-Thouless transition, leading to $T_{c}=\pi N_{B} /\left(2 A m_{B}\right)$ 
or equivalently $T_{c} / \epsilon_{F}=0.125\left(2 m c / d_{0}\right)$ for the Rashba SOC, where $N=k_{F}^{2} A /(2 \pi)$, with $A$ being the area.

\section{A. 3D Fermi gas with Rashba SOC}

In the molecular Bose gas limit, we obtain $d_{0}=$ $m V \sqrt{2 m|\mu|} /\left[8 \pi\left(2|\mu|-m \alpha^{2}\right)\right]$ for the time-dependent term and note that while the kinetic coefficient $c_{z z}=c_{z z}^{\text {intra }}=d_{0} /(2 m)$ has no interhelicity contribution, its in-plane element $c_{x x}=c_{y y}=c_{\perp} \quad$ is isotropic in the $x y$ plane with the following contributions: $c_{\perp}^{\text {intra }}=d_{0} /(2 m)-m V \sqrt{2 m} \alpha^{2} /\left[64 \pi \sqrt{|\mu|}\left(2|\mu|-m \alpha^{2}\right)\right]$ from the intrahelicity component and $c_{\perp}^{\text {inter }}=$ $[V \sqrt{2 m} /(64 \pi \sqrt{|\mu|})] \ln \left(2 m|\mu| / \sqrt{2 m|\mu|-m^{2} \alpha^{2}}\right)$ from the interhelicity one. Thus, while $m_{B}^{z z}=2 m$ is purely an intrahelicity contribution, we find $2 \mathrm{~m} / \mathrm{m}_{B \perp}^{\text {intra }}=$ $1-m \alpha^{2} /\left(2\left|\epsilon_{b}\right|+2 m \alpha^{2}\right)$ for the intrahelicity component and $2 m / m_{B \perp}^{\text {inter }}=\left[\left|\epsilon_{b}\right| /\left(2\left|\epsilon_{b}\right|+2 m \alpha^{2}\right)\right] \ln \left(1+m \alpha^{2} /\left|\epsilon_{b}\right|\right)$ for the interhelicity one, which are shown in Fig. 1. Using these analytic results, we conclude that $2 m / m_{B \perp}^{\text {intra }} \rightarrow\{1 / 2,0.653,1\}$ and $2 m / m_{B \perp}^{\text {inter }} \rightarrow\{0,0.181,0\}$ respectively, when $1 /\left(m \alpha a_{s}\right) \rightarrow\{-\infty, 0,+\infty\}$, such that the fraction of the interhelicity contribution to the pair mass is 0.217 at unitarity. Note that $T_{c} / \epsilon_{F} \rightarrow\{0.137,0.193,0.218\}$ for the same limits.

\section{B. 3D Fermi gas with Weyl SOC}

Similar to the Rashba case, here we obtain $d_{0}=$ $m V \sqrt{m}|\mu| /\left[4 \pi\left(2|\mu|-m \alpha^{2}\right)^{3 / 2}\right]$ for the time-dependent term and note that the kinetic coefficient $c_{x x}=c_{y y}=$ $c_{z z}=c$ is isotropic in all space with the following contributions: $\quad c^{\text {intra }}=d_{0} / 2 m-m V \sqrt{m} \alpha^{2} /[24 \pi(2|\mu|-$ $\left.m \alpha^{2}\right)^{3 / 2}$ ] from the intrahelicity component and $c^{\text {inter }}=[V \sqrt{m} /(12 \pi)]\left(1 / \sqrt{2|\mu|-m \alpha^{2}}-1 / \sqrt{2|\mu|}\right) \quad$ from the interhelicity one. Thus, we find $2 \mathrm{~m} / \mathrm{m}_{B}^{\text {intra }}=$ $1-2 m \alpha^{2} /\left(3\left|\epsilon_{b}\right|+3 m \alpha^{2}\right)$ for the intrahelicity component and $2 m / m_{B}^{\text {inter }}=4\left|\epsilon_{b}\right| /\left(3\left|\epsilon_{b}\right|+3 m \alpha^{2}\right)-(4 / 3)\left[\left|\epsilon_{b}\right| /\left(\left|\epsilon_{b}\right|+\right.\right.$ $\left.\left.m \alpha^{2}\right)\right]^{3 / 2}$ for the interhelicity one, which are again shown in Fig. 1. Using these analytic results, we also conclude that $2 m / m_{B}^{\text {intra }} \rightarrow\{1 / 3,2 / 3,1\}$ and $2 m / m_{B}^{\text {inter }} \rightarrow\{0,(2-$ $\sqrt{2}) / 3,0\}$, respectively, when $1 /\left(m \alpha a_{s}\right) \rightarrow\{-\infty, 0,+\infty\}$, such that the fraction of the interhelicity contribution to the pair mass is 0.226 at unitarity. Note again that $T_{c} / \epsilon_{F} \rightarrow\{0.0726,0.188,0.218\}$ for the same limits.

\section{2D Fermi gas with Rashba SOC}

In comparison to the 3D SOCs discussed above, here we obtain $d_{0}=\left\{m^{3} A \alpha /\left[4 \pi\left(2 m|\mu|-m^{2} \alpha^{2}\right)\right]\right\}[1 /(m \alpha)+$ $\left.\arctan \left(m \alpha / \sqrt{2 m|\mu|-m^{2} \alpha^{2}}\right) / \sqrt{2 m|\mu|-m^{2} \alpha^{2}}\right]$ for the coefficient of the time-dependent term, and note that the kinetic coefficient $c_{x x}=c_{y y}=c$ is isotropic in all space with the following contribution, $c^{\text {intra }}=$ $d_{0} / 2 m-\left\{m^{2} A \alpha /\left[16 \pi\left(2 m|\mu|-m^{2} \alpha^{2}\right)\right]\right\}[\alpha /(2|\mu|)+\arctan$ $\left.\left(m \alpha / \sqrt{2 m|\mu|-m^{2} \alpha^{2}}\right) / \sqrt{2 m|\mu|-m^{2} \alpha^{2}}\right]$, from the intrahelicity component and a similar contribution, $c^{\text {inter }}=[m A \alpha /$ $\left.(16 \pi|\mu|)] \arctan \left(m \alpha / \sqrt{2 m|\mu|-m^{2} \alpha^{2}}\right) / \sqrt{2 m|\mu|-m^{2} \alpha^{2}}\right]$, from the interhelicity component. Thus, we find a lengthy expression, $2 m / m_{B}^{\text {intra }}=1-$ $\left[m \alpha^{2} /\left(\left|\epsilon_{b}\right|+m \alpha^{2}\right)+\sqrt{m \alpha^{2} /\left|\epsilon_{b}\right|} \arctan \left(\sqrt{m \alpha^{2} /\left|\epsilon_{b}\right|}\right)\right] /[2+$

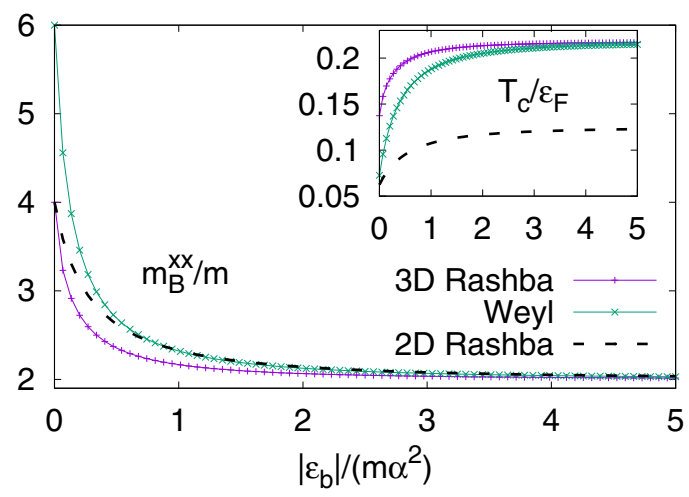

FIG. 2. The effective mass of the Cooper pairs $m_{B}^{x x}=d_{0} / c_{x x}$ and the critical SF transition temperature $T_{c} / \epsilon_{F}$ are shown for a molecular Bose gas. It is interesting to see that, since the interhelicity contribution is a slowly decaying function of $\left|\epsilon_{b}\right| /\left(m \alpha^{2}\right)$ as discussed in the main text, it accounts for a sizable fraction of the expected pair mass $m_{B}=2 m$ even in the $\left|\epsilon_{b}\right| \gg m \alpha^{2}$ limit as long as $\alpha \neq 0$. Thus, we conclude that the geometric contribution plays an integral role in the proper description of the molecular Bose gas. See also Refs. [40-45] for similar results.

$\left.2 \sqrt{m \alpha^{2} /\left|\epsilon_{b}\right|} \arctan \left(\sqrt{m \alpha^{2} /\left|\epsilon_{b}\right|}\right)\right]$, for the intrahelicity component and similarly $2 m / m_{B}^{\text {inter }}=$ $\left[\sqrt{m \alpha^{2}\left|\epsilon_{b}\right|} /\left(\left|\epsilon_{b}\right|+m \alpha^{2}\right) \arctan \left(\sqrt{m \alpha^{2} /\left|\epsilon_{b}\right|}\right)\right] /\left[1+\sqrt{m \alpha^{2} /\left|\epsilon_{b}\right|}\right.$ $\left.\arctan \left(\sqrt{m \alpha^{2} /\left|\epsilon_{b}\right|}\right)\right]$ for the interhelicity component, which are again shown in Fig. 1. Using these analytic results, we conclude that $2 m / m_{B}^{\text {intra }} \rightarrow\{1 / 2,1\}$ and $2 m / m_{B}^{\text {inter }} \rightarrow\{0,0\}$ in perfect correspondence with the 3D Rashba SOC discussed in Sec. V A, respectively, when $\left|\epsilon_{b}\right| /\left(m \alpha^{2}\right) \rightarrow\{0,+\infty\}$, and also that $T_{c} / \epsilon_{F} \rightarrow\{0.0625,0.125\}$ for the same limits.

In addition to illustrating all of the relevant limits mentioned above, one of the noteworthy revelations of Fig. 1 is that the peak values of the quantum-metric contribution coincide nearly with the locations of the unitarity in 3D systems. More importantly, Fig. 2 shows that since the interhelicity contribution is a slowly decaying function of $\left|\epsilon_{b}\right| /\left(m \alpha^{2}\right)$, it accounts for a sizable fraction of the expected pair mass $m_{B}=2 m$ even in the $\left|\epsilon_{b}\right| \gg m \alpha^{2}$ limit as long as $\alpha \neq 0$. Thus, our analysis suggests that the quantum-metric contribution is a nontrivial fraction of the pair mass in a fairly large parameter regime in the BCS-BEC crossover, making its experimental observation a real possibility with spin-orbit-coupled Fermi SFs.

\section{CONCLUSIONS}

In summary, here we showed that the quantum-metric contribution to the effective mass tensor of the Cooper pairs accounts for a sizable fraction of the pair mass in a surprisingly large parameter regime throughout the BCS-BEC crossover. This work reveals not only the physical origin of the governing role played by the quantum metric in the SF density tensor [14-16,29,31] but also hints at its plausible roles in many other observables including sound velocity, atomic compressibility, spin susceptibility, etc., all of which depend explicitly on the the pair mass. For instance, similar to the nonmonotonic evolution of the SF density, which is a direct consequence of 
the competition between the intrahelicity and interhelicity contributions at low temperatures [31], we expect nonmonotonic evolutions for those observables that are proportional to the kinetic coefficient $c_{i j}$ or equivalently to the inverse of the pair mass $1 / m_{B}$. As a final remark, it is worth pointing out that, even though most of our analysis in this paper is restricted to the spin-orbit-coupled Fermi SFs, our starting Hamiltonian density $H_{\mathbf{k}}$ is quite generic and may find direct applications in other two-band systems as well, e.g., in the contexts of quantum
spin-Hall effect [36] and superconductivity of Dirac electrons in graphene layers [37].

\section{ACKNOWLEDGMENTS}

The author thanks Atac Imamoglu for encouraging comments and acknowledges support from TÜBITTAK and the BAGEP award of the Turkish Science Academy.
[1] D. Xiao, M.-C. Chang, and Q. Niu, Berry phase effects on electronic properties, Rev. Mod. Phys. 82, 1959 (2010).

[2] X.-L. Qi and S.-C. Zhang, Topological insulators and superconductors, Rev. Mod. Phys. 83, 1057 (2011).

[3] R. Resta, The insulating state of matter: A geometrical theory, Eur. Phys. J. B 79, 121 (2011).

[4] J. Sinova, S. O. Valenzuela, J. Wunderlich, C. H. Back, and T. Jungwirth, Spin Hall effects, Rev. Mod. Phys. 87, 1213 (2015).

[5] A. Bansil, H. Lin, and T. Das, Colloquium: Topological band theory, Rev. Mod. Phys. 88, 021004 (2016).

[6] L. Lu, J. D. Joannopoulos, and M. Soljacic, Topological photonics, Nat. Photonics 8, 821 (2014).

[7] M. Wimmer, H. M. Price, I. Carusotto, and U. Peschel, Experimental measurement of the Berry curvature from anomalous transport, Nat. Phys. 13, 545 (2017).

[8] G. Jotzu, M. Messer, R. Desbuquois, M. Lebrat, T. Uehlinger, D. Greif, and T. Esslinger, Experimental realization of the topological Haldane model with ultracold fermions, Nature (London) 515, 237 (2014).

[9] L. Duca, T. Li, M. Reitter, I. Bloch, M. Schleier-Smith, and U. Schneider, An Aharonov-Bohm interferometer for determining Bloch band topology, Science 347, 288 (2015).

[10] M. Aidelsburger, M. Lohse, C. Schweizer, M. Atala, J. T. Barreiro, S. Nascimbéne, N. R. Cooper, I. Bloch, and N. Goldman, Measuring the Chern number of Hofstadter bands with ultracold bosonic atoms, Nat. Phys. 11, 162 (2015).

[11] S. Nakajima, T. Tomita, S. Taie, T. Ichinose, H. Ozawa, L. Wang, M. Troyer, and Y. Takahashi, Topological Thouless pumping of ultracold fermions, Nat. Phys. 12, 296 (2016)

[12] H.- I Lu, M. Schemmer, L. M. Aycock, D. Genkina, S. Sugawa, and I. B. Spielman, Geometrical Pumping with a Bose-Einstein Condensate, Phys. Rev. Lett. 116, 200402 (2016).

[13] J. P. Provost and G. Vallee, Riemannian structure on manifolds of quantum states, Commun. Math. Phys. 76, 289 (1980).

[14] L. Liang, T. I. Vanhala, S. Peotta, T. Siro, A. Harju, and P. Törmä, Band geometry, Berry curvature, and superfluid weight, Phys. Rev. B 95, 024515 (2017).

[15] S. Peotta and P. Törmä, Superfluidity in topologically nontrivial flat bands, Nat. Commun. 6, 8944 (2015).

[16] A. Julku, S. Peotta, T. I. Vanhala, D.-H. Kim, and P. Törmä, Geometric Origin of Superfluidity in the Lieb-Lattice Flat Band, Phys. Rev. Lett. 117, 045303 (2016).

[17] N. Marzari and D. Vanderbilt, Maximally localized generalized Wannier functions for composite energy bands, Phys. Rev. B 56, 12847 (1997).

[18] P. Zanardi, P. Giorda, and M. Cozzini, Information-Theoretic Differential Geometry of Quantum Phase Transitions, Phys. Rev. Lett. 99, 100603 (2007).
[19] F. D. M. Haldane, Geometrical Description of the Fractional Quantum Hall Effect, Phys. Rev. Lett. 107, 116801 (2011).

[20] T. Neupert, C. Chamon, and C. Mudry, Measuring the quantum geometry of Bloch bands with current noise, Phys. Rev. B 87, 245103 (2013).

[21] R. Roy, Band geometry of fractional topological insulators, Phys. Rev. B 90, 165139 (2014).

[22] T. S. Jackson, G. Moller, and R. Roy, Geometric stability of topological lattice phases, Nat. Commun. 6, 8629 (2015).

[23] Y. Gao, S. A. Yang, and Q. Niu, Field Induced Positional Shift of Bloch Electrons and Its Dynamical Implications, Phys. Rev. Lett. 112, 166601 (2014).

[24] Y. Gao, S. A. Yang, and Q. Niu, Geometrical effects in orbital magnetic susceptibility, Phys. Rev. B 91, 214405 (2015).

[25] M. Claassen, C. H. Lee, R. Thomale, X.-L. Qi, and T. P. Devereaux, Position-Momentum Duality and Fractional Quantum Hall Effect in Chern Insulators, Phys. Rev. Lett. 114, 236802 (2015).

[26] A. Srivastava and A. Imamoglu, Signatures of Bloch-Band Geometry on Excitons: Nonhydrogenic Spectra in TransitionMetal Dichalcogenides, Phys. Rev. Lett. 115, 166802 (2015).

[27] L.-K. Lim, J.-N. Fuchs, and G. Montambaux, Geometry of Bloch states probed by Stckelberg interferometry, Phys. Rev. A 92, 063627 (2015).

[28] F. Piéchon, A. Raoux, J.-N. Fuchs, and G. Montambaux, Geometric orbital susceptibility: Quantum metric without Berry curvature, Phys. Rev. B 94, 134423 (2016).

[29] M. Iskin, Berezinskii-Kosterlitz-Thouless transition in the timereversal-symmetric Hofstadter-Hubbard model, Phys. Rev. A 97, 013618 (2018).

[30] T. Ozawa, Steady-state Hall response and quantum geometry of driven-dissipative lattices, Phys. Rev. B 97, 041108(R) (2018).

[31] M. Iskin, Exposing the quantum geometry of spin-orbit coupled Fermi superfluids, arXiv:1711.07262.

[32] Z. Wu, L. Zhang, W. Sun, X.-T. Xu, B.-Z. Wang, S.-C. Ji, Y. Deng, S. Chen, X.-J. Liu, and J.-W. Pan, Realization of twodimensional spin-orbit coupling for Bose-Einstein condensates, Science 354, 83 (2016).

[33] W. Sun, B.-Z. Wang, X.-T. Xu, C.-R. Yi, L. Zhang, Z. Wu, Y. Deng, X.-J. Liu, S. Chen, and J.-W. Pan, Long-lived 2D SpinOrbit coupled Topological Bose Gas, arXiv:1710.00717.

[34] L. Huang, Z. Meng, P. Wang, P. Peng, S.-L. Zhang, L. Chen, D. Li, Q. Zhou, and J. Zhang, Experimental realization of a two-dimensional synthetic spin-orbit coupling in ultracold Fermi gases, Nat. Phys. 12, 540 (2016).

[35] Z. Meng, L. Huang, P. Peng, D. Li, L. Chen, Y. Xu, C. Zhang, P. Wang, and J. Zhang, Experimental Observation of a Topological Band Gap Opening in Ultracold Fermi Gases with 
Two-Dimensional Spin-Orbit Coupling, Phys. Rev. Lett. 117, 235304 (2016).

[36] B. A. Bernevig, T. L. Hughes, and S. C. Zhang, Quantum spin Hall effect and topological phase transition in HgTe quantum well, Science 314, 1757 (2006).

[37] N. B. Kopnin and E. B. Sonin BCS Superconductivity of Dirac Electrons in Graphene Layers, Phys. Rev. Lett. 100, 246808 (2008).

[38] M. Drechsler and W. Zwerger, Crossover from BCSsuperconductivity to Bose-condensation, Ann. Phys. 504, 15 (1992).

[39] C. A. R. Sá de Melo, M. Randeria, and J. R. Engelbrecht, Crossover from BCS to Bose Superconductivity: Transition Temperature and Time-Dependent Ginzburg-Landau Theory, Phys. Rev. Lett. 71, 3202 (1993).

[40] M. Iskin and A. L. Subaşı, Quantum phases of atomic Fermi gases with anisotropic spin-orbit coupling, Phys. Rev. A 84, 043621 (2011).
[41] L. Jiang, X.-J. Liu, H. Hu, and H. Pu, Rashba spin-orbitcoupled atomic Fermi gases, Phys. Rev. A 84, 063618 (2011).

[42] L. He and X.-G. Huang, BCS-BEC Crossover in 2D Fermi Gases with Rashba Spin-Orbit Coupling, Phys. Rev. Lett. 108, 145302 (2012).

[43] L. He and X.-G. Huang, BCS-BEC crossover in threedimensional Fermi gases with spherical spin-orbit coupling, Phys. Rev. B 86, 014511 (2012).

[44] J. P. Vyasanakere and V. B. Shenoy, Collective excitations, emergent Galilean invariance, and boson-boson interactions across the BCS-BEC crossover induced by a synthetic Rashba spin-orbit coupling, Phys. Rev. A 86, 053617 (2012).

[45] T. Yamaguchi and Y. Ohashi, Proposed method to realize the $p$-wave superfluid state using an $s$-wave superfluid Fermi gas with a synthetic spin-orbit interaction, Phys. Rev. A 92, 013615 (2015). 